\title{
Fungus gnats (Diptera: Sciaroidea) of the Gemer region (Central Slovakia): Part 1 - Bolitophilidae, Diadocidiidae, Ditomyiidae and Keroplatidae
}

\author{
Jan Ševčík \& Olavi Kurina
}

\begin{abstract}
Fungus gnats (Diptera: Sciaroidea) of the Gemer region (central Slovakia): Part 1 - Bolitophilidae, Diadocidiidae, Ditomyiidae and Keroplatidae. - Čas. Slez. Muz. Opava (A), 60: 11-23, 2011.

Abstract: A total of 17 species of Bolitophilidae, 4 species of Diadocidiidae, 2 species of Ditomyiidae and 25 species of Keroplatidae are recorded from the Gemer region in central Slovakia. The material was obtained mainly in the years 2008-2010 by means of Malaise traps and by individual collecting by the authors. Five species, Bolitophila spinigera Edwards, 1925, Antlemon servulum (Walker, 1837), Orfelia nigricornis (Fabricius, 1805), Macrocera maculata Meigen, 1818 and Macrocera zetterstedti Lundström, 1914, are recorded from Slovakia for the first time. The occurrence of Asindulum nigrum Latreille, 1805 in Slovakia is confirmed.
\end{abstract}

Key words: Insecta, Diptera, Sciaroidea, new records, faunistics, Slovakia, Europe

\section{Introduction}

Families of Sciaroidea (Diptera), so called fungus gnats, belong to one of the most species-rich groups of insects, with more than 5000 described species worldwide, but probably a similar number of species still await description. More than 1000 species of these families occur in Europe. In the recent checklist, the following numbers of species are reported from Slovakia for particular families: Bolitophilidae 19 (Košel \& Ševčík 2009a), Diadocidiidae 5 (Ševčík \& Košel 2009a), Ditomyiidae 4 (Košel \& Ševčík 2009b), Keroplatidae 45 (Ševčík \& Košel 2009b) and Mycetophilidae 445 (Ševčík \& Košel 2009c).

The adults are common in the undergrowth of forests, especially in shaded places alongside streams, in cavities under tree roots and overhanging stream banks, but sometimes also in other habitats such as meadows, caves and wetlands. The larvae are mostly mycophagous, in some groups predaceous, and the biology of many species still remains unknown. For a review of Czech and Slovak species of Diptera associated with fungi and their fungus hosts see Ševčík (2010).

The families of Sciaroidea had not been systematically studied in the Gemer region up to 2008 when the All Taxa Biodiversity Inventories (ATBI) project started. Only Košel \& Laštovka (1997) published data on several species of Exechiini (Mycetophilidae) from caves in the Slovakian Karst National Park (= Slovenský kras NP). A few species found during our faunal survey within ATBI as new to the fauna of Slovakia have already been published (see Ševčík 2009; Kurina \& Ševčík 2011).

This paper is the first of two devoted to the fauna of fungus gnats in the Gemer region. The second forthcoming paper will include the species of Mycetophilidae.

\section{Material and methods}

The list of species below is based mainly on the material collected recently $(2008-2010)$ in the Gemer region by means of Malaise traps and sweep netting, identified by the authors and now deposited in IZBE (all material collected by OK), JSOC and SMOC (all the other material). Three Malaise traps (Figs 1-3) were operated in the area, all in the Muránska planina NP:

1. PR Fabova Hola, spring area in a young spruce forest, 1230 m, 15.v.-25.viii.2009, J. Ševčík leg.

2. NPR Javorníková, limestone canyon with deciduous (mainly beech) forests, 414 m, 27.v. -5.vi.2009, O. Kurina leg.

3. NPR Hrdzavá, limestone canyon with deciduous forest, 570 m, 1.v.- 7.x.2010, J. Ševčík leg.

The material from the year 2001 collected with a Malaise trap by L. Vidlička at Paseky near Tisovec (Muránska planina NP) is also included (see Vidlička 2004). 

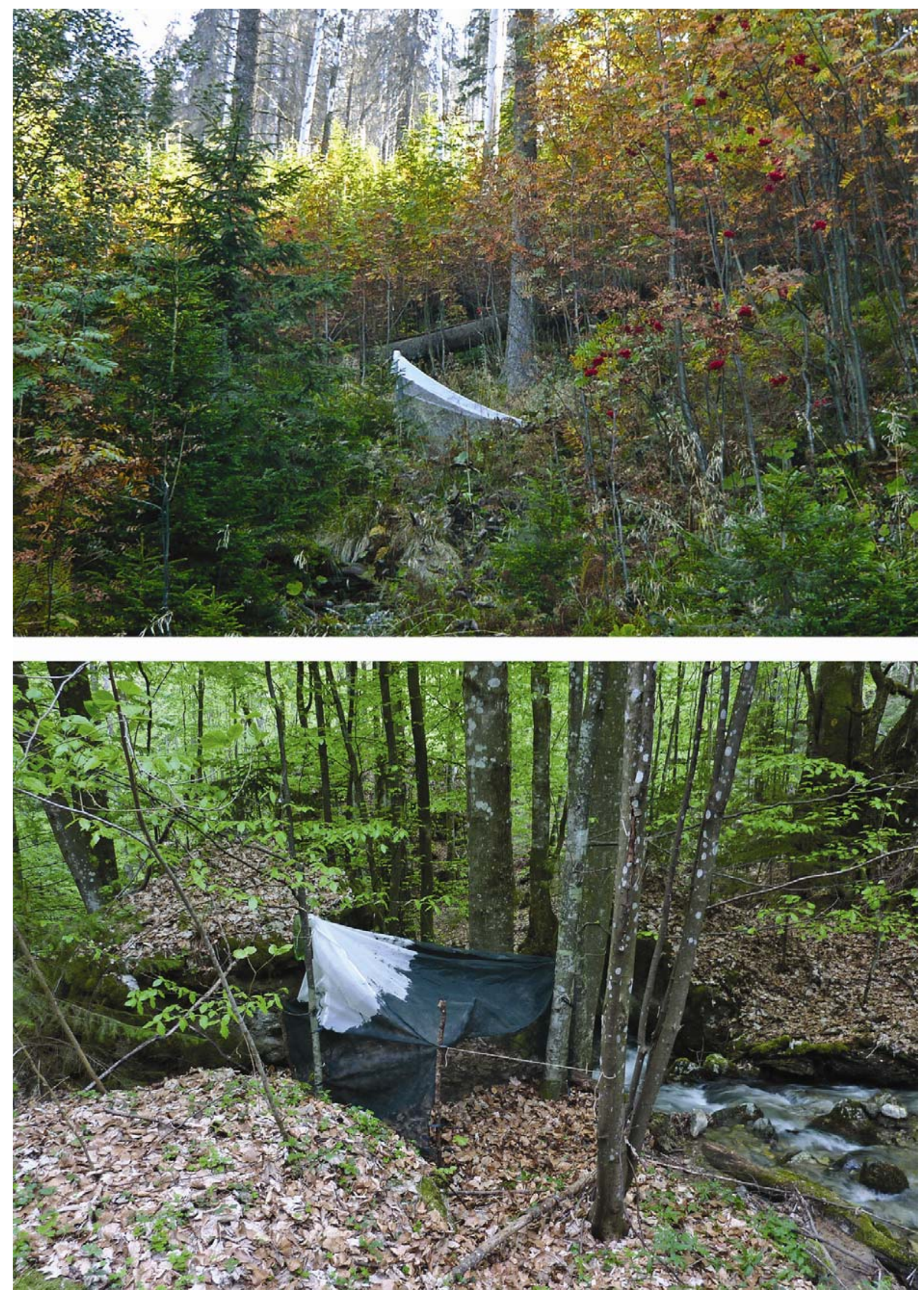

Figs 1 and 2: Malaise traps at Fabova Hola (1) and Hrdzavá dolina (2) in Muránska planina National Park (photo by J. Ševčík) 

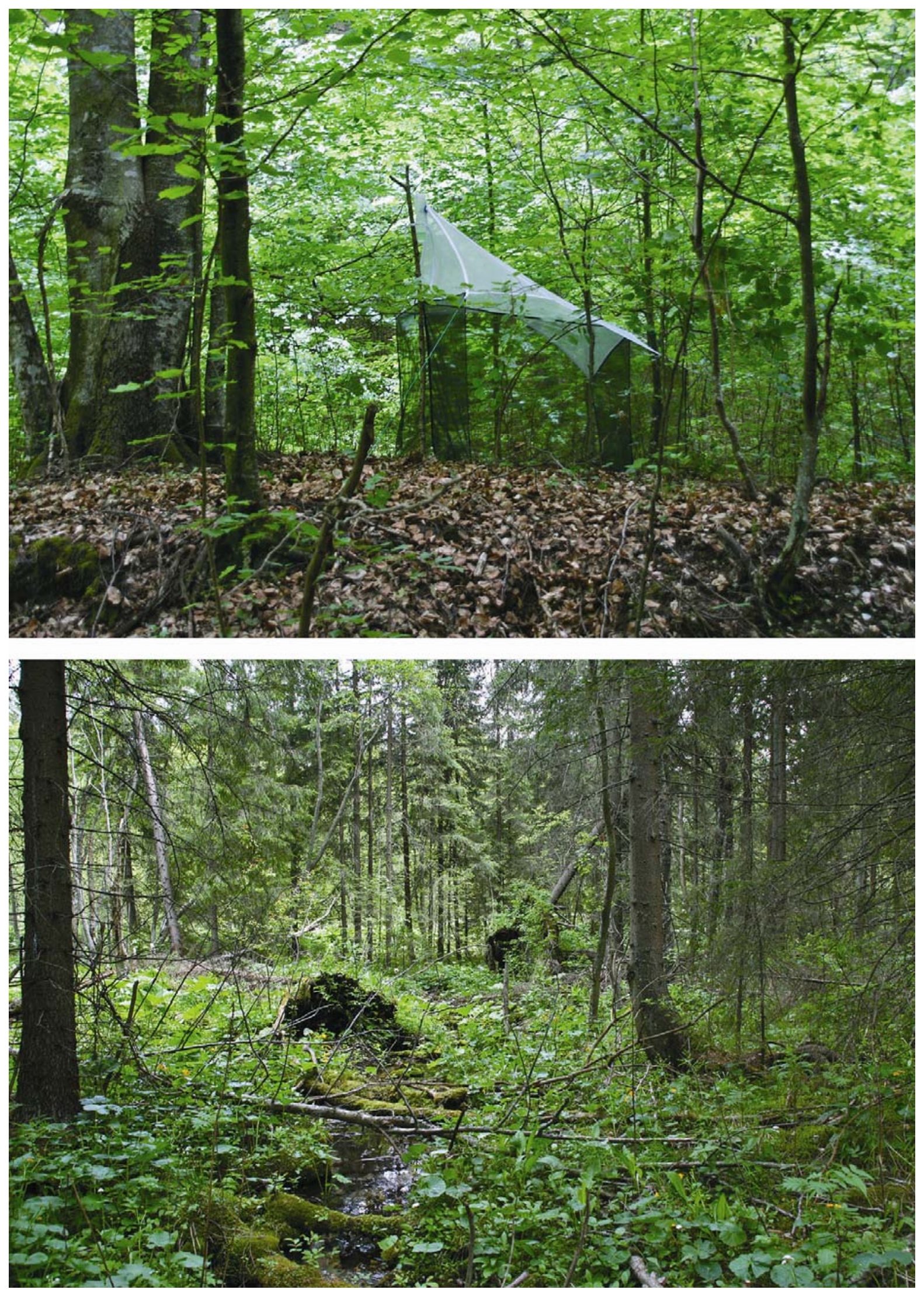

Figs 3 and 4: Malaise trap in Javorníková dolina (3); Hnilecká jelšina in Slovenský raj National park (4). Photo by O. Kurina. 

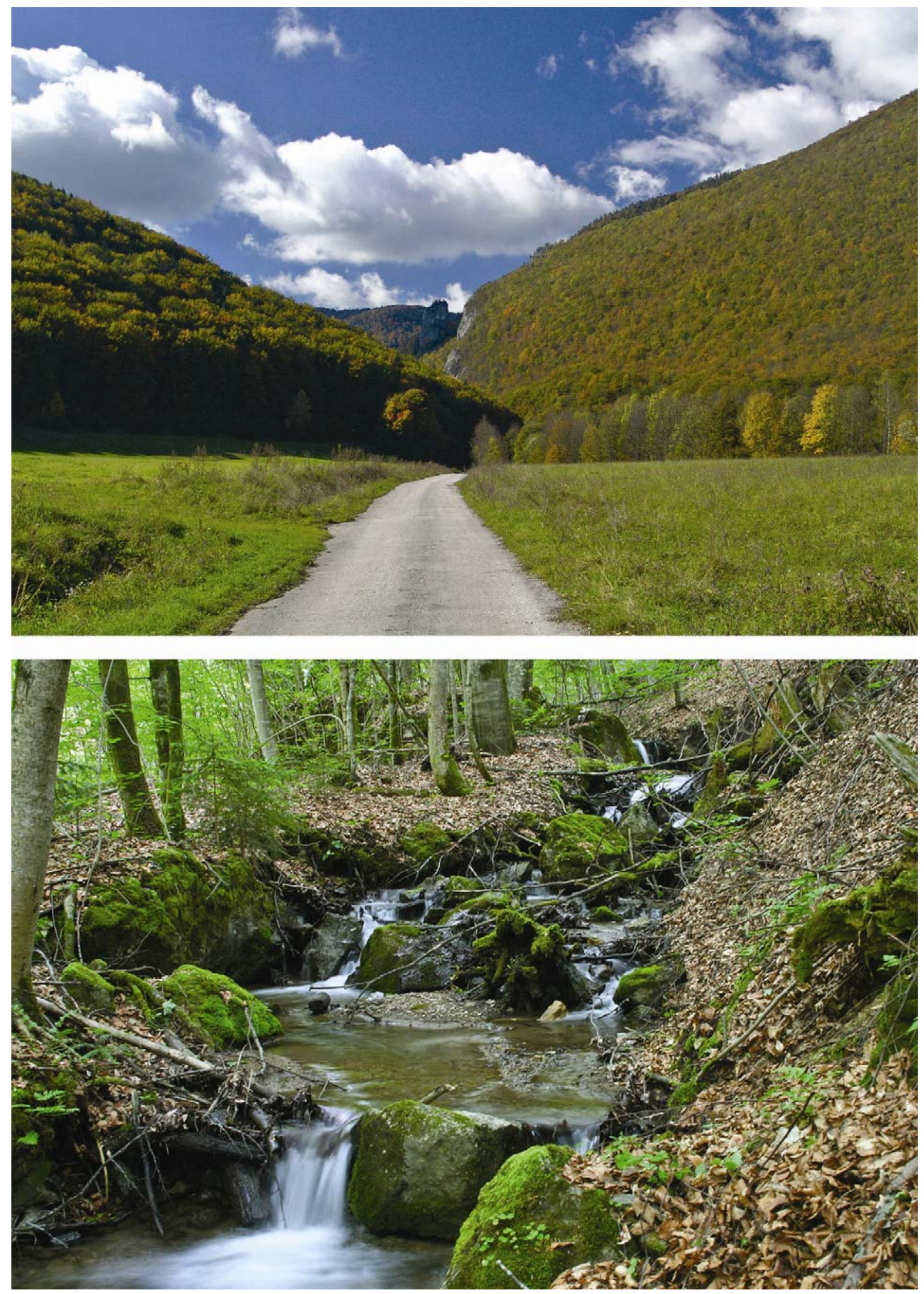

Figs 5 and 6: Hrdzavá dolina National Nature Reserve in Muránska planina National park (5, photo by J. Ševčík); stream banks in the Hrdzavá dolina Valley (6, photo by O. Kurina) 

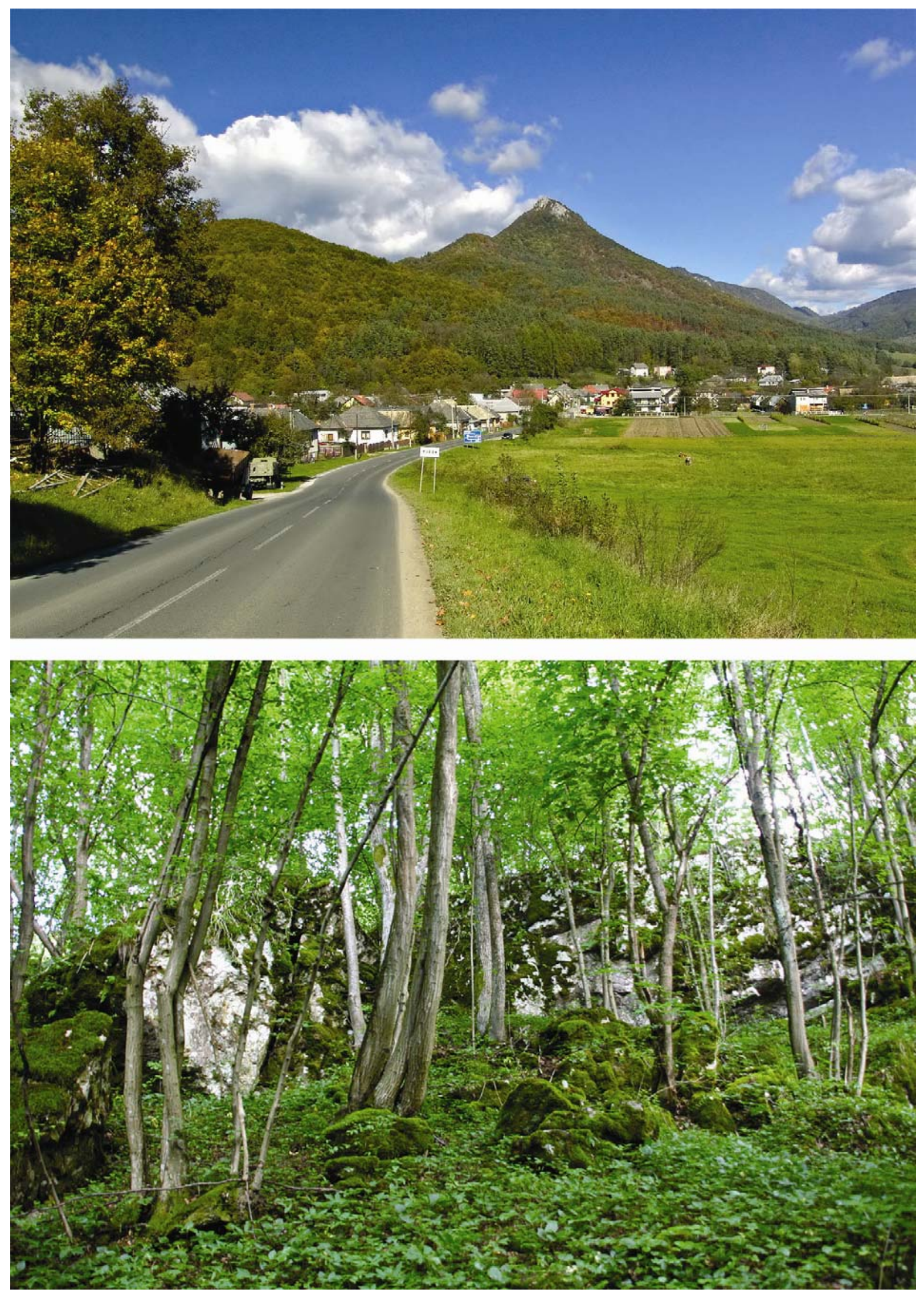

Figs 7 and 8: Muránska planina National park around the village Muráň (7, photo by J. Ševčík); surroundings of the Silická Ladnica Cave in Slovenský kras National park (8, photo by O. Kurina) 
The nomenclature used here principally follows the Fauna Europaea database, version 2.4 (Chandler 2011).

In the species list, the collecting method is indicated only for Malaise traps (as MT), otherwise the material was collected by sweeping.

Abbreviations: $*=$ new records for Slovakia; IZBE $=$ Institute of Agricultural and Environmental Science, Estonian University of Life Sciences, former Institute of Zoology and Botany, NP = National Park, NPP = National Nature Monument, NPR = National Nature Reserve, $\mathrm{PR}=$ Nature Reserve, $\mathrm{m}=$ male, $\mathrm{f}=$ female, $\mathrm{OK}=$ Olavi Kurina leg., JŠ = Jan Ševčík leg., MT = Malaise trap, JSOC = Jan Ševčík, Ostrava (personal collection), SMOC $=$ Slezské zemské muzeum, Opava, Czech Republic, spec. $=$ specimens.

\section{Study area}

The Gemer area is considered here to cover the territory of three national parks in central Slovakia Muránska planina NP, Slovenský raj NP and Slovenský kras NP. Most of the material presented in this paper was collected in the frame of the All Taxa Biodiversity Inventories project (ATBI, see www.atbi.eu) organized by Workpackage 7 (WP7) of the "European Distributed Institute of Taxonomy" (EDIT, see www.etaxonomy.eu). All the three national parks represent well-preserved karst areas with both montane and thermophilous species, including several that are endemic. The results of the species inventory within the ATBI project are also available on the Internet page http://www.atbi.eu/gemer/. For selected collecting localities see Figures 4-8.

\section{Survey of species}

\section{BOLITOPHILIDAE}

\section{Bolitophila (B.) austriaca (Mayer, 1950)}

A rare Palaearctic species. Recorded from Slovakia by Košel (2004) and Ševčík (2009b).

Localities. NP Muránska planina: road to Kl'ak, 1211m, 26.v.2009, 7 spec. (m/f); Ladová jama na Muráni, vegetation at cave entrance, $1002 \mathrm{~m}, 26 . v .2009,35 \mathrm{spec} .(\mathrm{m} / \mathrm{f})$, vegetation in spruce forest, 1178m, 2.vi.2009, 3 spec. (m/f); Javorníková dolina - middle part, 468m, 27.v.2009, 3 f; Cigánka, small karst cave, 844m 30.v.2009, 1 f; Havrania dolina, along a waterless rivulet in spruce forest, 828m 28.v.2009, 7 spec. (m/f). NP Slovenský kras: Silická Ladnica, 567m, vegetation around cave entrance, 3.vi.2009, 1 f (all OK).

\section{Bolitophila (B.) basicornis (Mayer, 1951)}

A rare Palaearctic species. Ševčík (2004b) recorded it from Poloniny NP as new to Slovakia and recently also from Pol'ana Mts (Ševčík 2009b). It is treated as a vulnerable (VU) species in the Czech Republic (Ševčík 2005).

Localities. NP Muránska planina: road to Kl'ak, 1211m, 26.v.2009, 14 spec. (m/f); Hrdzavá dolina, 726m, 29.v.2009, 1 f; Ladová jama na Muráni, vegetation at cave entrance, 1002m, 26.v.2009, 4 spec. (m/f); Maretkiná, along a narrow rivulet in mixed forest, 1002m, 26.v.2009, 5 spec. (m/f); Vel'ká lúka, along a narrow ravine in mixed forest, 878m, 26.v.2009, 3 spec. (m/f); Havrania dolina, 828m, 28.v.2009, 4 spec. (m/f); Zlatnica, deciduous forest along a stream, 788m, 28.v.2009, 3 f. NP Slovenský raj: Hranovnické pleso, vegetation in mixed forest, 800m, 2.vi.2009, $1 \mathrm{~m} 1 \mathrm{f}$; Kopanec, vegetation in spruce forest, 997m, 2.vi.2009, $6 \mathrm{f}($ all OK).

\section{Bolitophila (B.) cinerea Meigen, 1818}

A common Palaearctic species. Larvae develop in various agarics (see Ševčík 2010). Recently recorded from Slovakia (Pol'ana Mts) by Ševčík (2009b).

Localities. NP Muránska planina: around Bobačka, Fabova Hola, Dolina Hronca, road to Kl'ak, Šarkanica, Hrdzavá dolina, Ladová jama na Muráni, Javorníková, Maretkiná, Poludnica, Cigánka, Vel'ká lúka, Lopušná, Zlatnica, Mokrá Polana, Trsteník. NP Slovenský kras: NPR Zádielská tiesňava. NP Slovenský raj: Javorina Mt., Hnilecká jelšina, Hranovnické pleso, Kopanec (JŠ, OK).

Sampling dates: v.-ix.

\section{Bolitophila (B.) tenella Winnertz, 1863}

A rather rare Palaearctic species. Recently recorded from Slovakia (Pol'ana Mts) by Ševčík (2009b).

Localities. NP Muránska planina: road to Kl'ak, 1211m, 26.v.2009, 10 m; Ladová jama na Muráni, vegetation at cave entrance, 1002m, 26.v.2009, $1 \mathrm{~m}$; Vel'ká lúka, mixed forest, 878m, 26.v.2009, 2 m (all OK); NPP Bobačka, along brook in deciduous forest, 910m, 1.v.2010, $1 \mathrm{~m}$ (JŠ). 
*Bolitophila (B.) spinigera Edwards, 1925

A rare European species. This is the first record from Slovakia.

Localities. NP Muránska planina: road to Kl'ak, 1211m, 26.v.2009, 1 m, spruce forest with Vaccinium myrtillus (OK).

Bolitophila (C.) bimaculata Zetterstedt, 1838

A rare European species. Recently recorded from Slovakia (Tatra Mts) by Ševčík (2010).

Localities. NP Muránska planina: NPR Hrdzavá, 520m, 1.v.2010, 1 m, along brook in deciduous forest (JŠ).

Bolitophila (C.) dubia Siebke, 1863

A rather rare but widespread Palaearctic species. Recorded from Slovakia (Poloniny NP) only by Martinovský (1995).

Localities. NP Muránska planina: road to Kl'ak, 1211m, 26.v.2009, 4 f; Ladová jama na Muráni, vegetation at cave entrance, 1002m, 26.v.2009 3 spec. (m/f); Vel'ká lúka, mixed forest, 878m, 26.v.2009, 2 (m/f); Lopušná, vegetation in spruce forest, 1061m, 28.v.2009, $1 \mathrm{~m}$; Zlatnica, deciduous forest along a stream and a track, 788m, 28.v.2009, 1 m. NP Slovenský raj: Javorina Mt., 934m, 4.vi.2009, 1 f; Hranovnické pleso, 894m, 2.vi.2009, $1 \mathrm{~m}$ (all OK).

Bolitophila (C.) edwardsiana Stackelberg, 1969

A rare Eurosiberian species. Recorded from Slovakia (Poloniny NP) only by Martinovský (1995).

Localities. NP Slovenský raj: Hranovnické pleso, vegetation in mixed forest, 894m, 2.vi.2009, 1 m (OK).

Bolitophila (C.) fumida Edwards, 1941

A rare Palaearctic species. Recently recorded from Slovakia (Pol'ana Mts) by Ševčík (2009b).

Locality. NP Muránska planina: Dolina Hronca, 760m, 6.ix.2008, 1 m (JŠ). NP Slovenský raj: Hnilecká jelšina, 925m, 4.vi.2009, $1 \mathrm{~m}$ (both OK).

Bolitophila (C.) glabrata Loew, 1869

A rare Palaearctic species. Recently recorded from Slovakia (Malá Fatra and Pol'ana Mts) by Ševčík (2002, 2009b).

Localities. NP Slovenský kras: NPR Drieňovec, 500m, along waterless brook in a hornbeam forest, 5.ix.2008, $1 \mathrm{~m}$ (JŠ).

Bolitophila (C.) hybrida (Meigen, 1804)

A widespread Holarctic species. Its larvae develop in Paxillus involutus and P. filamentosus (cf. Ševčík 2010). Recently recorded from Slovakia (Horná Orava Mts) by Ševčík (2010).

Localities. NP Slovenský kras: NPR Drieňovec, $500 \mathrm{~m}$, along waterless brook in a hornbeam forest, 5.ix.2008, 1 m; NPR Zádielská tiesňava, 480m, 5.ix.2008 1 m (JŠ).

Bolitophila (C.) ingrica Stackelberg, 1969

A rare Palaearctic species. Ševčík (2004) recorded it as new to Slovakia from Poloniny NP (Stužica Reserve) and subsequently also from Pol'ana Mts (Ševčík 2009b).

Localities. NP Muránska planina: NPR Hrdzavá, 520m, 14.v.2009, 1 m, 1.v.2010, 6 m; around NPP Bobačka, 910m, 1.v.2010, 3 m (JŠ), Vel'ká lúka, 878m, 26.v.2009, 1 f; Havrania dolina, along a waterless rivulet in spruce forest, 828m, 28.v.2009, $1 \mathrm{f}(\mathrm{OK})$.

Bolitophila (C.) maculipennis Walker, 1836

A rare Palaearctic species. Recently recorded from Slovakia (Pol'ana Mts) by Ševčík (2009b).

Localities. NP Muránska planina: Vel'ká lúka, 878m, 26.v.2009, 1 m. NP Slovenský raj: Hnilecká jelšina, 925m, 4.vi.2009, $1 \mathrm{~m}$ (both OK).

Bolitophila (C.) modesta Lackschewitz, 1937

A rare Palaearctic species. Recently recorded from Slovakia (Pol'ana Mts) by Ševčík (2009b).

Localities. NP Muránska planina: Vel'ká lúka, 878m, 26.v.2009, 1 f; Lopušná, vegetation in spruce forest, $1061 \mathrm{~m}, 28 . v .2009,9$ f (both OK).

Bolitophila (C.) nigrolineata Landrock, 1912

A rare but widespread Palaearctic species. Recently recorded from Slovakia (Pol'ana Mts) by Ševčík (2009b). Locality. NP Muránska planina: Maretkiná, mixed forest, 1002m, 26.v.2009, 1 f (OK); NPP Bobačka, along brook in deciduous forest, 910m, 1.v.2010, 2 m (JŠ). NP Slovenský raj: Hranovnické pleso, 800m, 2.vi.2009, $2 \mathrm{~m}$; Kopanec, 997m, vegetation in spruce forest, 2.vi.2009, $1 \mathrm{f}$ (both OK).

Bolitophila (C.) occlusa Edwards, 1913

A common Palaearctic species. Larvae develop in Postia polypores (see Ševčík 2010). Recorded for the first time from Slovakia by Martinovský (1995).

Localities. NP Muránska planina: Ladová jama na Muráni, Hrdzavá, Javorníková, Maretkiná, Havrania dolina (JŠ, OK). NP Slovenský raj: Hnilecká jelšina (OK). 


\section{Bolitophila (C.) sp. indet.}

This species is figured by Zaitzev (1994) under the most probably misidentified name B. modesta (see Ševčík 2010). Its identity is not clear at present, but it is reliably known from central Slovakia (Pol'ana Mts) and northern Europe (Jostein Kjærandsen pers. comm.).

Locality. NP Muránska planina: PR Fabova Hola, spring area in young spruce forest, 1230m, 15.v.200916.vi.2009, $1 \mathrm{~m}$ (JŠ, MT).

\section{DIADOCIDIIDAE}

\section{Diadocidia (A.) trispinosa Polevoi, 1996}

A very rare boreomontane species similar to the Nearctic D. borealis Coquillett, 1900. It is known from northern and central Europe. Recently recorded from Slovakia (Pol'ana Mts) by Ševčík (2009b).

Locality. NP Muránska planina: Hrdzavá dolina, 726m, 29.v.2009, 2 m, along brook (OK).

\section{Diadocidia (A.) valida Mik, 1874}

A rare but widely distributed European species. Recorded from Slovakia by Laštovka \& Matile (1972) and Martinovský (1995).

Locality. NP Muránska planina: PR Fabova Hola, 1230m, 16.vi.-25.viii.2009, 2 m 1 f(JŠ, MT).

\section{Diadocidia (D.) ferruginosa (Meigen, 1830)}

A very common Palaearctic species. Recently recorded from Slovakia (Pol'ana Mts) by Ševčík (2009b).

Localities. NP Muránska planina: Dolina Dudlavky, Javorníková, Havrania dolina, Hrdzavá, Mokrá Pol'ana, Poludnica, Šarkanica, Šiance, Vel'ká lúka, Zlatnica. NP Slovenský kras - Krásnohorská cave, Drieňovec, Zádielská tiesňava. NP Slovenský raj: Hnilecká jelšina, Kopanec.

Sampling dates: v.-ix.

\section{Diadocidia (D.) spinosula Tollet, 1948}

A rather common Palaearctic species. Recently recorded from Slovakia (Pol'ana Mts) by Ševčík (2009b). Localities. NP Muránska planina: Dolina Dudlavky, road to Kl'ak, Hrdzavá, Javorníková, Poludnica, Cigánka, Vel'ká lúka, Lopušná, Trstenik, Zlatnica. NP Slovenský kras: Zádielská tiesňava. NP Slovenský raj: Mt. Javorina, Hnilecká jelšina, Kopanec.

Sampling dates: v.-ix.

\section{DITOMYIIDAE}

\section{Ditomyia fasciata (Meigen, 1818)}

A common European species. Larvae develop in various species of tree fungi. Recently recorded from Slovakia (Poloniny NP and Pol'ana Mts) by Ševčík (2009b, 2010).

Locality. NP Slovenský kras: NPR Drieňovec, 500m, 5.ix.2008, 1 m, along waterless brook in a hornbeam forest (JŠ).

\section{Symmerus annulatus (Meigen, 1830)}

A locally common Eurosiberian species.

Localities. NP Muránska planina: NPR Hrdzavá, 570m, 1.v.2010 - 28.vi.2010, 3 m (JŠ, MT); Šiance, 656m, 30.v.2009, $3 \mathrm{~m}$, vegetation in deciduous forest (OK).

\section{KEROPLATIDAE Keroplatinae}

\section{*Antlemon servulum (Walker, 1837)}

A rare species, recorded from Great Britain and Ireland, France, Germany, Hungary, Italy, Switzerland, the Ukraine and the Czech Republic (Chandler 2011). This is the first record from Slovakia.

Locality. NP Slovenský raj: NPR Stratená, 27.ix.2009, 1 m (JŠ).

\section{Asindulum nigrum Latreille, 1805}

A rare species, recorded from several localities in Europe. The first finding from Slovakia by Straka \& Majzlan (2007) has remained doubtful but the current record confirms occurrence of the species. Locality. NP Muránska planina; Paseky near Tisovec, 14.-17.viii.2001, 1 f (L'. Vidlička, MT).

\section{Cerotelion racovitzai Matile \& Burghele-Balacesco, 1969}

A rather rare species, recorded from several localities in eastern and central Europe, also in Azerbajdan and Iran. In Slovakia also recorded from Poloniny NP (Martinovský 1995) and Pol'ana Mts (Ševčík 2009b).

Locality. NP Muránska planina: NPR Hrdzavá, 520m, along brook in deciduous forest, 3.ix.2008, 1m (JŠ). 
Cerotelion striatum (Gmelin, 1790)

Widely distributed in Europe and in the Near East. Recorded from Slovakia (Poloniny NP and Pol'ana Mts) by Martinovský (1995) and Ševčík (2009b).

Locality. NP Muránska planina: NPR Hrdzavá, 570m, 28.vi.2010 - 26.vii.2010 1 m (JŠ, MT).

\section{Isoneuromyia semirufa (Meigen, 1818)}

A widely distributed Palaearctic species. Recorded for the first time from Slovakia (Poloniny NP) by Martinovský (1995).

Locality. NP Slovenský kras: NPR Drieňovec, 500m, 5.ix.2008, 1 m, along waterless brook in a hornbeam forest (JŠ).

\section{Keroplatus testaceus Dalman, 1818}

A widespread and relatively common Palaearctic species. Larvae live on the surface of various tree fungi (e.g. Trametes gibbosa, see Ševčík 2010).

Localities. NP Muránska planina: NPR Hrdzavá, 570m, 28.vi.2010 - 26.vii.2010 $1 \mathrm{~m}$ (JŠ, MT); Paseky near Tisovec, 19.-22.vi.2001, 1 m (L. Vidlička, MT). NP Slovenský kras: NPR Zádielská tiesňava, 480m, 5.ix.2008, $1 \mathrm{~m}$, along brook in limestone canyon (JŠ).

\section{Neoplatyura modesta (Winnertz, 1863)}

A rather rare European species. Biology unknown. Recorded for the first time from Slovakia (Pol'ana Mts) by Ševčík (2009b).

Locality. Muránska planina NP: Paseky near Tisovec, 10.-14.viii.2001, 1 m (L'. Vidlička, MT).

\section{Orfelia discoloria (Meigen, 1818)}

A rather rare European species. Recorded for the first time from Slovakia (Poloniny NP) by Martinovský (1995).

Locality. Muránska planina NP: Paseky near Tisovec, 10.-14.viii.2001, 2 m (L. Vidlička, MT).

\section{Orfelia fasciata (Meigen, 1804)}

A rather common European species. Recently recorded from Slovakia (Poloniny NP, Pol'ana Mts) by Martinovský (1995) and Ševčík (2009b).

Locality. NP Slovenský kras: Silická Ladnica, 567m, vegetation around cave entrance, 3.vi.2009, $1 \mathrm{~m}, 1 \mathrm{f}$ $(\mathrm{OK})$.

\section{Orfelia gruevi Bechev, 2002}

This species was described from Bulgaria and subsequently recorded from Greece, so this is the northernmost record of the species (cf. Ševčík 2009a).

Locality. NP Muránska planina: NPR Cigánka, 930m, vegetation along the ruins of castle, 4.ix.2008, $1 \mathrm{~m}$ (JŠ, Ševčík 2009a).

\section{Orfelia nemoralis (Meigen, 1818)}

A common European species, mainly in steppe and forest-steppe habitats.

Locality. Muránska planina NP: Paseky near Tisovec, 19.-22.vi.2001, 3 m, 4.-6.vii.2001, 1 m (L'. Vidlička, MT).

\section{*Orfelia nigricornis (Fabricius, 1805)}

A rare European species. Although this species was recorded for the first time from Slovakia by Straka \& Majzlan (2007), their record was not accepted by Ševčík \& Košel (2009b). New record for Slovakia.

Locality. Muránska planina NP: Paseky near Tisovec, 10.-14.viii.2001, 1 m (L. Vidlička, MT).

\section{Platyura marginata Meigen, 1804}

A locally common Palaearctic species. Recently recorded from Slovakia (Poloniny NP, Pol'ana Mts) by Martinovský (1995) and Ševčík (2009b).

Localities. NP Muránska planina: NPR Hrdzavá, 570m, 1.v.2010-28.vi.2010, 2 m (JŠ, MT); Javorníková dolina, 414m, 27.v. - 5.vi.2009, 2 m (OK, MT); Paseky near Tisovec, 19.-22.vii.2001, 1 m (L. Vidlička, MT).

\section{Pyratula zonata (Zetterstedt, 1855)}

An uncommon Palaearctic species. Recently recorded from Slovakia (Poloniny NP, Pol'ana Mts) by Martinovský (1995) and Ševčík (2009b).

Localities.: NP Muránska planina: Šiance, 656m, vegetation in deciduous forest, 30.v.2009, $1 \mathrm{~m}$. NP Slovenský kras: Silická Ladnica, 505m, vegetation around cave entrance, 3.vi.2009, 2 m; Krásnohorská jaskyňa, 312m, along a stream and at a cave entrance, 3.vi.2009, $4 \mathrm{~m}$ (both OK).

Urytalpa dorsalis (Staeger, 1840)

A rather common European species, until recently referred to as $U$. ochracea (Meigen, 1818), see Kjærandsen et al. (2009). From Slovakia (Poloniny NP, Pol'ana Mts) recently recorded by Martinovský (1995) and Ševčík (2009b).

Locality. NP Muránska planina: Predná Hora, 836m, 2.vi.2009, 1 female (OK). 


\section{Macrocerinae}

\section{Macrocera angulata Meigen, 1818}

A common European species. Recently recorded from Slovakia (Poloniny NP, Pol'ana Mts) by Martinovský (1995) and Ševčík (2009b).

Locality. NP Muránska planina: Dolina Hronca, 760m, 6.ix.2008, 1 m (JŠ).

\section{Macrocera fasciata Meigen, 1804}

A relatively common Palaearctic species. Recently recorded from Slovakia (Poloniny NP, Pol'ana Mts) by Martinovský (1995) and Ševčík (2009b).

Localities. NP Muránska planina: Javorníková dolina, 414m, 27.v.2009, 1 f (OK); NPR Poludnica, 460m, 4.ix.2008, 1 m. NP Slovenský kras: NPR Drieňovec, 500m, 5.ix.2008, 1 m (both JŠ).

\section{Macrocera fastuosa Loew, 1869}

A very rare European species. Recorded from Slovakia for the first time by Ševčík (2009b). The male from Muránska planina has several macrotrichia on the wing membrane and the central fascia is not distinctly reaching above $\mathrm{R} 4+5$ (see Fig. 10) but we consider this as a mere intraspecific variation until more material is collected.

Locality. NP Muránska planina: NPR Hrdzavá, 570m, 26.vii.2010-7.x.2010, 1 m (JŠ, MT).

\section{*Macrocera maculata Meigen, 1818}

A rather rare European species, new to Slovakia. The opportunity is also taken here to publish one more recent record from Pol'ana Mts overlooked by Ševčík (2009b): Havranie skaly, 5.vii.2006, 1 m (JŠ).

Locality. NP Muránska planina: Javorníková dolina: middle part, moist canyon with logs and trunks, 468m, 27.v.2009, $1 \mathrm{f}(\mathrm{OK})$.

\section{Macrocera parva Lundström, 1914}

A rare montane Palaearctic species. Recently recorded from Slovakia (Poloniny NP, Pol'ana Mts) by Martinovský (1995) and Ševčík (2009b).

Localities. NP Muránska planina: PR Fabova Hola, spring area in young spruce forest, 1230m, 15.v.200916.vi.2009, $1 \mathrm{~m}$ (JŠ, MT); road to Kl'ak, spruce forest with Vaccinium myrtillus, 1211m, 26.v.2009, $1 \mathrm{~m}$; Ladová jama na Muráni, vegetation in spruce forest, 1178m, 2.vi.2009, 2 m (both OK).

\section{Macrocera phalerata Meigen, 1818}

A common Palaearctic species. Recently recorded from Slovakia (Poloniny NP, Pol'ana Mts) by Martinovský (1995) and Ševčík (2009b).

Locality. NP Muránska planina: NPR Poludnica, along small brook in deciduous forest, 460m, 4.ix.2008, 1 $\mathrm{m}(\mathrm{J} \breve{S})$.

\section{Macrocera pilosa Landrock, 1917}

A rare European species. Recorded from Slovakia (NP Slovenský kras, Zádielská dolina) for the first time by Martinovský (2001).

Localities. NP Muránska planina, NPR Poludnica, top plateau, light deciduous forest, 942m, 16.v.2009, 6 m; NPP Bobačka, along brook in deciduous forest, 910m, 1.v.2010, $1 \mathrm{~m}$ (both JŠ); Vel'ká lúka, mixed forest, 878m, 26.v.2009, 2 f (OK).

\section{Macrocera stigma Curtis, 1837}

A rather common Palaearctic species. Recently recorded from Slovakia (Poloniny NP, Pol'ana Mts) by Martinovský (1995) and Ševčík (2009b).

Locality. NP Muránska planina: PR Fabova Hola, 15.v.2009-16.vi.2009, 1 m (JŠ, MT).

\section{Macrocera stigmoides Edwards, 1925}

A common Palaearctic species. Recently recorded from Slovakia (Poloniny NP, Pol'ana Mts) by Martinovský (1995) and Ševčík (2009b).

Localities. NP Muránska planina: Cigánka, Hrdzavá. NP Slovenský kras: Drieňovec. Sampling dates: v.-ix.

*Macrocera zetterstedti Lundström, 1914

A rare montane species (see Fig. 9), hitherto known only from northern Europe and from the Czech Republic (Ševčík 2001). New for Slovakia.

Localities. NP Muránska planina: Hrdzavá dolina, along a ravine: Sphagnum, Vaccinium, Pinus mugo, 726m, 29.v.2009, 3 m; road to Kl'ak, spruce forest with Vaccinium myrtillus, 1211m, 26.v.2009, $1 \mathrm{~m}$ (both OK). 

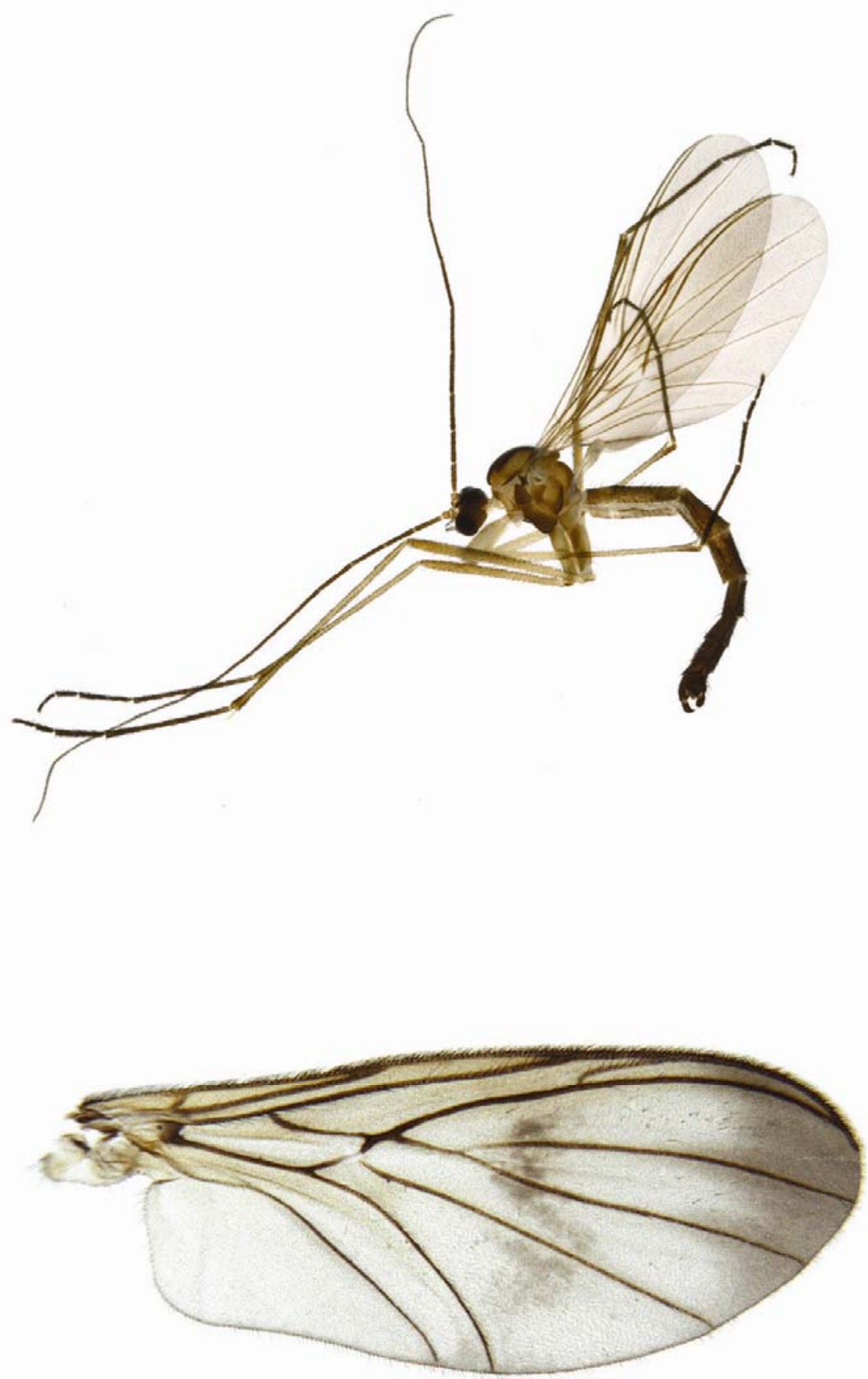

Figs 9 and 10: habitus of Macrocera zetterstedti Lundström, 1914 (9, photo by O. Kurina); wing of the specimen of Macrocera fastuosa Loew, 1869 from Muránska planina (10, photo by J. Ševčík) 


\section{Conclusions}

A total of 17 species of Bolitophilidae, 4 species of Diadocidiidae, 2 species of Ditomyiidae and 25 species of Keroplatidae have been recorded from the Gemer region up to the present, including 5 species recorded here as new to Slovakia (Bolitophila spinigera, Antlemon servulum, Orfelia nigricornis, Macrocera maculata, Macrocera zetterstedti). The occurrence of Asindulum nigrum in Slovakia is confirmed. The very rare Orfelia gruevi has already been recorded by Ševčík (2009a) from the region (surroundings of the Murán Castle) which represents the northernmost known locality of this species, otherwise known only from Bulgaria and Greece. A few additional species may be considered rare and noteworthy, viz. Bolitophila nigrolineata, Diadocidia trispinosa, Orfelia discoloria, Orfelia nigricornis, Macrocera fastuosa, Macrocera parva and Macrocera pilosa.

Gemer region thus belongs to the three best-studied and most species-rich areas in Slovakia with regard to the fauna of fungus gnats, together with Poloniny National Park (= Bukovské vrchy Mts, see Martinovský 1995) in easternmost Slovakia and Pol'ana Mts (see Ševčík 2009b) in central Slovakia. The communities of fungus gnats in the Gemer Region demonstrate the uniqueness and high nature conservation value of the ecosystems in the area.

Acknowledgements: We are grateful to the administration of the ATBI-Gemer project, particularly to Katarína Necpálová and Eduard Stloukal, and to the staff of the Administration of the Muránska planina National Park for their help and hospitality during our study visits to the area. JŠ thanks Ms. Tereza Hasíková for her pleasant company and help during the field work. JŠ was partly supported by the Czech Science Foundation (grant No. 206/08/1500) and by the IGS 201103 grant of the Silesian Museum in Opava. OK was also partially supported by Estonian Science Foundation grant 7558.

\section{References}

Chandler P. (2011): Fauna Europaea: Mycetophilidae. In Jong H. de (ed.): Fauna Europaea: Diptera, Nematocera. Fauna Europaea Version 2.4, http://www.faunaeur.org.

Kjærandsen, J., Martinsson, S., Hedmark, K. \& Evenhuis, N. L. (2009): On the genus Urytalpa Edwards (Diptera: Keroplatidae) in the Nordic and Nearctic regions, with fixation of a new type species and a key to world males.- Zootaxa, 2160: 29-50.

Košel V. (2004): Parietal Diptera in caves of the Belianske Tatry Mts (Slovakia, the Western Carpathians) I. Introduction and species spectrum.- Acta Facultatis Ecologiae, 12, Suppl. 1: 69-73.

Košel V. \& Laštovka P. (1997): Faunistic records from the Czech and Slovak republics: Diptera. Mycetophilidae. In Rozkošný R. \& Vaňhara J. (eds.): Dipterologica bohemoslovaca. Vol. 8.- Folia Facultatis Scientiarum Naturalium Universitatis Masarykianae Brunensis, Biologia 95: 214-215.

Košel V. \& Ševčík J. (2009a): Bolitophilidae Malloch, 1917. In Jedlička L., Kúdela M. \& Stloukalová V. (eds): Checklist of Diptera of the Czech Republic and Slovakia. Electronic version 2. <http://zoology.fns.uniba.sk/diptera2009> + CD-ROM: ISBN 978-80-969629-4-5.

- (2009b): Ditomyiidae Edwards, 1921. In Jedlička L., Kúdela M. \& Stloukalová V. (eds): Checklist of Diptera of the Czech Republic and Slovakia. Electronic version 2. $<$ http://zoology.fns.uniba.sk/diptera2009> + CD-ROM: ISBN 978-80-969629-4-5.

Kurina O. \& Ševčík J. (2011): Three new species of Docosia Winnertz from central and southern Europe (Diptera: Mycetophilidae).- Zootaxa, 2810: 26-36.

Martinovský J. (1995): Mycetophilidae, p. 50-61. In Roháček J., Starý J., Martinovský J. \& Vála M. (eds): Diptera Bukovských vrchov. Diptera of the Bukovské hills. Humenné, 232 pp.

- (2001): New records on little-known western Palaearctic Macrocera Meigen, with a description of M. jonica sp.n. from Greece (Diptera: Keroplatidae).-Acta Universitatis Carolinae Biologica 45: 109-114.

Straka V. \& Majzlan O. (2007): Dvojkrídlovce (Diptera) PR Kopáč pri Bratislave. Pp. 233-260. In: Majzlan, O. (ed.) Príroda ostrova Kopáč. Fytoterapia OZ, Bratislava, 287 pp.

Ševčík J. (1999): Fifty species of fungus gnats (Diptera: Mycetophilidae) new for the Czech Republic and/or Slovakia, including a new species of Allodia Winnertz.- Casopis Slezského zemského muzea, Opava (A) 48 : 97-105.

- (2001): New records of Diadocidiidae, Keroplatidae and Mycetophilidae (Diptera: Sciaroidea) from the Czech Republic.- Časopis Slezského zemského muzea, Opava (A) 50: 159-169.

- (2002): New records of Bolitophilidae, Keroplatidae and Mycetophilidae (Diptera) from Slovakia.- Biologia, 
Bratislava 57: 198, 212.

- (2004a): New data on Sciaroidea (Diptera) from the Czech and Slovak Republics, with descriptions of seven new species of Mycetophilidae.- Časopis Slezského zemského muzea, Opava (A) 53: 49-74.

- (2004b): Diptera associated with fungi in the Poloniny National Park (Bukovské vrchy Mts., East Slovakia).Folia Facultatis Scientiarum Naturalium Universitatis Masarykianae Brunensis, Biologia 109: 293-304.

- (2009a): Two new species and other new records of fungus gnats (Diptera: Mycetophilidae and Keroplatidae) from Slovakia and the Czech Republic.- Časopis Slezského zemského muzea, Opava (A) 58: 55-60.

- (2009b): Bolitophilidae (pp. 49-51), Diadocidiidae (p. 51), Ditomyiidae (pp. 52-53), Keroplatidae (pp. 53-56). In Roháček J. \& Ševčík J. (eds): Diptera of the Pol'ana Protected Landscape Area - Biosphere Reserve (Central Slovakia). Zvolen. 340 pp.

- (2010): Czech and Slovak Diptera associated with fungi. Slezské zemské muzeum, Opava. 112 pp.

Ševčík J. \& Košel V. (2009a): Diadocidiidae Winnertz, 1863. In Jedlička L., Kúdela M. \& Stloukalová V. (eds): Checklist of Diptera of the Czech Republic and Slovakia. Electronic version 2. $<$ http://zoology.fns.uniba.sk/diptera2009> + CD-ROM: ISBN 978-80-969629-4-5.

- (2009b): Keroplatidae Rondani, 1856. In Jedlička L., Kúdela M. \& Stloukalová V. (eds): Checklist of Diptera of the Czech Republic and Slovakia. Electronic version 2. <http://zoology.fns.uniba.sk/diptera2009> + CD-ROM: ISBN 978-80-969629-4-5.

- (2009c): Mycetophilidae Newman, 1834. In Jedlička L., Kúdela M. \& Stloukalová V. (eds): Checklist of Diptera of the Czech Republic and Slovakia. Electronic version 2. $<$ http://zoology.fns.uniba.sk/diptera2009> + CD-ROM: ISBN 978-80-969629-4-5.

Vidlička L. (2004): Siet’okrídlovce (Neuroptera) Muránskej planiny I. - lokalita Paseky.- Reussia 1, Suppl. 1: 241-244.

Zaitzev A. I. (1994): Fungus gnats of the fauna of Russia and adjacent regions. Part. I. Nauka, Moscow. 288 pp.

Authors' addresses: Jan Ševčík, University of Ostrava, Chittussiho 10, CZ-710 00 Ostrava \& Silesian Museum, Tyršova 1, CZ-746 01 Opava, Czech Republic. E-mail: sevcikjan@hotmail.com Olavi Kurina, Institute of Agricultural and Environmental Sciences, Estonian University of Life Sciences, Riia st 181, 51014 Tartu, Estonia. E-mail: olavi.kurina@emu.ee

\section{Dvoukřídlí nadčeledi Sciaroidea (Diptera) regionu Gemer (stř̌ední Slovensko): Část 1 - Bolitophilidae, Diadocidiidae, Ditomyiidae a Keroplatidae}

Celkem 17 druhů čeledi Bolitophilidae, 4 druhy Diadocidiidae, 2 druhy Ditomyiidae a 25 druhů Keroplatidae je zaznamenáno z regionu Gemer na středním Slovensku. Materiál byl získán zejména v letech 2008-2010 pomocí Malaiseho pastí a individuálním sběrem autorů. Pět druhů, a to Bolitophila spinigera Edwards, 1925, Antlemon servulum (Walker, 1837), Orfelia nigricornis (Fabricius, 1805), Macrocera maculata Meigen, 1818 a Macrocera zetterstedti Lundström, 1914, je zaznamenáno ze Slovenska poprvé. Potvrzen je zde výskyt druhu Asindulum nigrum Latreille, 1805. 


\title{
Book offer
}

\section{Czech and Slovak Diptera associated with fungi}

\author{
Jan Ševčík \\ $16.5 \times 24 \mathrm{~cm}, 112$ pages , 35 colour photographs \\ Published by Slezské zemské muzeum, 2010 \\ Printed by Retis Group s.r.o., Krnov, Czech Republic \\ ISBN 978-80-86224-84-8 \\ Price (plus postage and bank transfer fees) 8 Euro
}

This work summarizes data on 242 species of Diptera belonging to 27 families reared by the author from 248 species of macrofungi and myxomycetes collected in the Czech and Slovak Republics in the years 1998 - 2010. Most species recorded belong to the family Mycetophilidae (99 species), followed by the families Phoridae (21 spp.), Cecidomyiidae (19 spp.), Drosophilidae (15 spp.), Bolitophilidae (10 spp.), Platypezidae (10 spp.), Muscidae (10 spp.), Sphaeroceridae (8 spp.) and Heleomyzidae (6 spp.). The other families were represented by 5 or less species. For each species a list of hitherto known fungus hosts in the Czech and Slovak Republic is given, including the previous literature records. A systematic list of host fungi with associated insect species is also provided. The first record of any host fungus is given for Mycetophila ostentanea Zaitzev, 1998, Mycetophila sordida van der Wulp, 1874 and Sceptonia flavipuncta

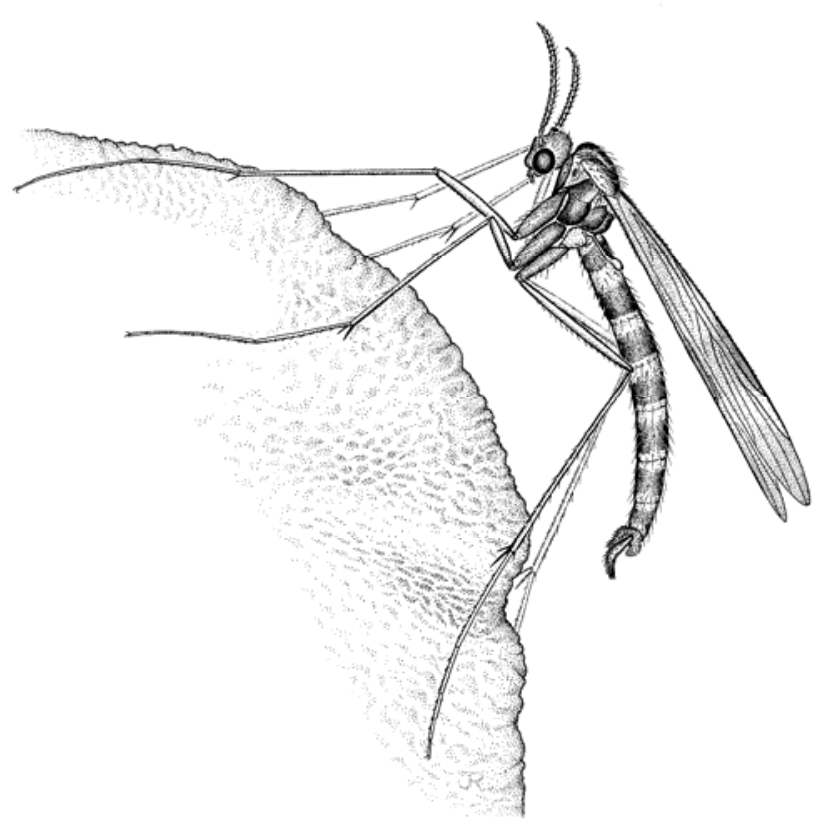
Edwards, 1925 (all Mycetophilidae). Comments are also made on larval parasitoids reared from Mycetophilidae and Phoridae.

"This substantial work is an update of the 2006 publication Diptera associated with fungi in the Czech and Slovak Republics by the same author. That first edition was the result of rearing from fungi over the previous 8 years and included records of 188 species of Diptera in 26 families from 188 species of fungi and 1 species of myxomycetes. The present work includes all of that data plus more recent results and the new totals are 242 species of Diptera from 245 species of fungi and 3 species of myxomycetes. It includes rearing records of three species of an additional fly family, Scatopsidae. ... A comprehensive account is given of what is known about the biology of each of the species reared and this is followed by an interesting discussion of the range of flies associated with fungi and their host selection. The account of hymenopterous parasitoids reared during this study and the systematic list of the host fungi with their associated Diptera are useful additions to the work. The accuracy of identification of the flies, parasitoids and fungus hosts was assured by involvement of specialists. ... The work is well illustrated by drawings and photographs, showing adults and larvae in association with their hosts as well as some of their habitats. Several of these and two plates of host fungi are new for this edition." (From the review by P. J. Chandler)

Orders should be sent to: Slezské zemské muzeum, Tyršova 1, 74601 Opava, Czech Republic or by e-mail to david.fiser@szmo.cz 